\title{
Samambaias e Licófitas do Parque Estadual do Guartelá, PR, Brasil
}

\author{
Cássio Michelon ${ }^{1,2}$ e Paulo Henrique Labiak ${ }^{1}$
}

Recebido: 20.07.2012; aceito: 25.02.2013

\begin{abstract}
Ferns and Lycophytes from Parque Estadual do Guartelá, Paraná State, Brazil). This study deals with the survey of ferns and lycophythes from Parque Estadual do Guartelá, Paraná State, Brazil. The area is characterized by a mosaic of distinct vegetational and geological formations. We found 164 species, distributed in 18 families and 59 genera. It was evident that the vegetational/geological configuration is a determining factor on the habitat preference of the species. Cheilanthes goyazensis and Phlegmariurus badinianus are the first records for the Paraná State, while Lindsaea stricta is the second record for the State. Beside those, Anemia ferruginea and Cyathea myriotricha constitute the third record for the State. We also found eight new records for the Segundo Planalto Paranaense. We conclude that the environmental heterogeneity is directly related to the high floristic richness found in the park.
\end{abstract}

Key words: campos gerais, canyon, rupestrian field, sandstone

RESUMO -(Samambaias e Licófitas do Parque Estadual do Guartelá, PR, Brasil). Este estudo trata do levantamento das espécies de samambaias e licófitas do Parque Estadual do Guartelá, Paraná, área caracterizada por um intricado mosaico de formações vegetacionais e geológicas. Foram encontradas 164 espécies, distribuídas em 18 famílias e 59 gêneros. Ficou evidente que a configuração fitofisionomica/geológica é um fator determinante na preferência habitats das espécies encontradas. Cheilanthes goyazensis e Phlegmariurus badinianus são o primeiro registro para o Estado do Paraná, já Lindsaea stricta é o segundo registro para o Estado, enquanto Anemia ferruginea e Cyathea myriotricha constituem o terceiro registro para o Estado. Além dessas, oito espécies são novos registros para o Segundo Planalto Paranaense. Concluímos que a heterogeneidade ambiental da área esta diretamente relacionada com a elevada riqueza florística local.

Palavras-chave: arenito, campos gerais, campo rupestre, canyon

\section{Introdução}

No início do Segundo Planalto Paranaense, limitado a leste pela Escarpa Devoniana, principia a região denominada Campos Gerais, definida por Maack (1981) como uma zona fitogeográfica natural que inclui áreas de campo, matas de galeria, capões de araucária e refúgios de cerrado. Este tipo de formação geológica/vegetacional tem demonstrado resguardar uma diversidade de samambaias bastante rica, com algumas espécies sendo recentemente descritas para a região (Schwartsburd et al. 2007, Schwartsburd \& Labiak, 2008, Christenhuzs et al. 2009). De qualquer forma, estudos envolvendo esses grupos no Segundo Planalto Paranaense ainda são escassos (Sakagami 2006, Schwartsburd \& Labiak 2007).

Estudos realizados no Parque Estadual do Guartelá trataram predominantemente da geologia e geomorfologia (e.g. Melo 2000, Retzlaf et al. 2006, Hornes et al. 2008); e nos estudos envolvendo a flora local (e.g. Takeda et al. 1996, Ziller \& Hatschbach 1996, Carmo 2006), pouca ou nenhuma menção às samambaias e licófitas é feita.

Considerando a importância biológica encontrada nessa região e a carência de estudos com ênfase nos grupos estudados, o presente trabalho pretende suprir parte da lacuna existente no conhecimento da diversidade ali resguardada, ampliando a distribuição geográfica de várias espécies e fornecendo uma lista completa para essa Unidade de Conservação do Estado do Paraná.

\section{Material e métodos}

O Parque Estadual do Guartelá (PEG), inserido na Área de Preservação Ambiental da Escarpa

1. Universidade Federal do Paraná, Departamento de Botânica, Curso de Pós-graduação em Botânica, Caixa Postal 19031, 81531-980 Curitiba, PR, Brasil

2. Autor para correspondência: cassio.michelon@gmail.com 
Devoniana, está localizado no município de Tibagi, na região dos Campos Gerais do Segundo Planalto Paranaense, abrangendo 798,97 hectares (IAP 2002). A temperatura média anual é de $18{ }^{\circ} \mathrm{C}$, enquanto a precipitação média anual encontra-se entre 1.400 a $1.600 \mathrm{~mm}$ e a umidade relativa anual varia entre $80 \%$ e $85 \%$ (UEPG 2003). A altitude varia de 770 a 1.100 m s.n.m. (medições realizadas com GPS Garmin eTrex Vista $\mathrm{H})$.

A principal litologia observada na área é o Arenito Furnas, que apresenta erosão diferencial causada pelas variações estruturais da rocha, acarretando no relevo ruiniforme característico do PEG. O relevo é bastante diversificado, variando de suave-ondulado a extremamente acidentado, com as escarpas chegando a mais de $100 \mathrm{~m}$ de altura (Diedrichs 1995). Justamente devido a essas características, são observadas diferentes coberturas vegetais, todas associadas à geologia e geomorfologia local. Embora a fitofisionomia predominante seja o campo higrófilo (seco) não arborizado, são também observados campos sujos, campos hidromórficos (úmidos), vegetação rupestre, pequenas áreas de Cerrado, capões de floresta e a floresta ripária do rio Iapó.

Expedições mensais de coleta foram realizadas durante o período de março de 2010 a setembro de 2011, nas quais cerca de 450 espécimes foram coletados. Além dessas coletas, foram realizadas consultas às coleções dos herbários UPCB, MBM e HUPG. A identificação do material coletado foi realizada por consulta a trabalhos de revisão, floras regionais, aos herbários da região e a especialistas. Os materiais coletados foram depositados no herbário UPCB e duplicatas enviadas, principalmente, aos herbários MBM, SP e UC.

Os dados da distribuição geográfica das espécies foram obtidos a partir de trabalhos de flora, revisões e herbários consultados. Os padrões de distribuição utilizados foram: Circum-Antártica, África/América, Neotropical, América do Sul e Brasil.

Para a determinação dos ambientes preferenciais das espécies foram considerados os seguintes padrões vegetacionais: capões de floresta, florestas de galeria, floresta ripária do rio Iapó, ambientes ripários de lajeado, campos secos, campos úmidos, campos litólicos e ambientes antropizados.

Para as análises de similaridade florística entre os tipos de formações do PEG foi elaborada uma matriz "espécie" $\times$ "formação vegetacional" com os dados binários do tipo presença/ausência. A partir dessa matriz foi gerado um dendrograma de agrupamento (UPGMA) (figura 1) utilizando como índice de semelhança o Índice de Jaccard. Para a análise de similaridade florística entre o PEG e outras 21 áreas de interesse (Floresta Atlântica brasileira) foi elaborada uma matriz "espécie" × "área", com os dados binários do tipo presença/ausência, a partir da qual foi realizada análise de ordenação do tipo NMDS (figura 2). Nessa análise não houve diferenças significativas entre os índices de Jaccard, Bray-Curtis e a Distância Euclidiana. De qualquer forma, optou-se pelo Bray-Curtis devido melhor visualização gráfica dos resultados. Além disso, este parece ser o índice mais robusto utilizado atualmente neste tipo de análise (Minchin 1987, Melo \& Hepp 2008). Ambas as análises foram processadas pelo programa PAST (Hammes et al. 2001).

O sistema de classificação da vegetação adotado foi o proposto por Veloso et al. (1991).

O sistema de classificação utilizado para as famílias de samambaias foi o de Smith et al. (2008) e as de licófitas foi aquele proposto por Christenhusz et al. (2011). Os nomes dos autores de táxons foram verificados em Tropicos (2012).

\section{Resultados e Discussão}

Foram encontradas 164 espécies (148 samambaias e 16 licófitas), distribuídas em 18 famílias e 59 gêneros (tabela 1). Este número representa $37 \%$ - $42 \%$ da flora de samambaias e licófitas do Paraná e aproximadamente $13 \%$ do Brasil (Zuloaga et al. 2008, Forzza et al. 2010). As famílias mais expressivas foram Polypodiaceae (25 espécies), Pteridaceae (22 espécies), Dryopteridaceae (18 espécies) e Hymenophyllaceae (16 espécies), que juntas representam aproximadamente $48 \%$ das espécies encontradas no PEG. Já os gêneros mais representativos foram Thelypteris (13 espécies) e Asplenium e Blechnum (12 espécies cada).

As samambaias e licófitas representam aproximadamente $20 \%$ da flora vascular registrada no PEG (834 espécies no total, somando as espécies registradas por Carmo (2006) e Mancinelli (dados não publicados). Esta taxa corresponde de duas a quatro vezes o esperado para áreas continentais (5\% - 10\%) (Moran 2008), demonstrando possível carência no estudo das angiospermas do Parque. De qualquer forma, mesmo considerando que a flora local de angiospermas esteja subamostrada, o PEG abriga um número bastante representativo de samambaias e licófitas, tornando-o importante sítio para a conservação dos grupos estudados. 
Em relação aos ambientes preferenciais, fica evidente a importância da configuração fitofisionomica/ geológica característica do PEG para a diversidade dos grupos estudados, já que 79 espécies $(48,2 \%)$ foram observadas exclusivamente em um tipo de ambiente. A heterogeneidade ambiental encontrada no PEG, também registrada em outros estudos (Klein \& Hatschbach 1971, Carmo 2006, Schwartsburd \& Labiak 2007), é característica marcante da região dos Campos Gerais e a possível explicação para a elevada riqueza encontrada na área.

Das 164 espécies, 137 foram verificadas nas formações florestais (capões de floresta, florestas de galeria e floresta ripária do rio Iapó), 53 em formações campestres (ambientes ripários de lajeado, campos secos, campos úmidos e campos rupestres) e 25 em ambos os tipos de formações. Somente uma espécie (Cyathea atrovirens) foi observada em todos os ambientes encontrados no PEG.
As formações rochosas representam importante abrigo para as espécies encontradas, uma vez que 82 espécies $(50,3 \%)$ ocorrem nesses ambientes e 20 delas são exclusivas. Dentre as espécies restritas aos afloramentos destacam-se Anemia sp. nov., Blechnum asplenioides, Blechnum laevigatum, Cheilanthes goyazensis, Cyathea myriotricha $\mathrm{e}$ Jamesonia areniticola, além de diversas espécies de Hymenophyllaceae.

Apesar das formações campestres abrigarem pequeno número de espécies (comparando a outros estudos, como Schwartsburd \& Labiak 2007, e em relação às formações florestais do PEG), estas mostraram-se como importantes registros para o Estado do Paraná.

Em relação a cada tipo de formação vegetacional encontrada na área de estudo, temos:

Capões de floresta: os capões isolados em meio às áreas de campos mostraram-se bastante

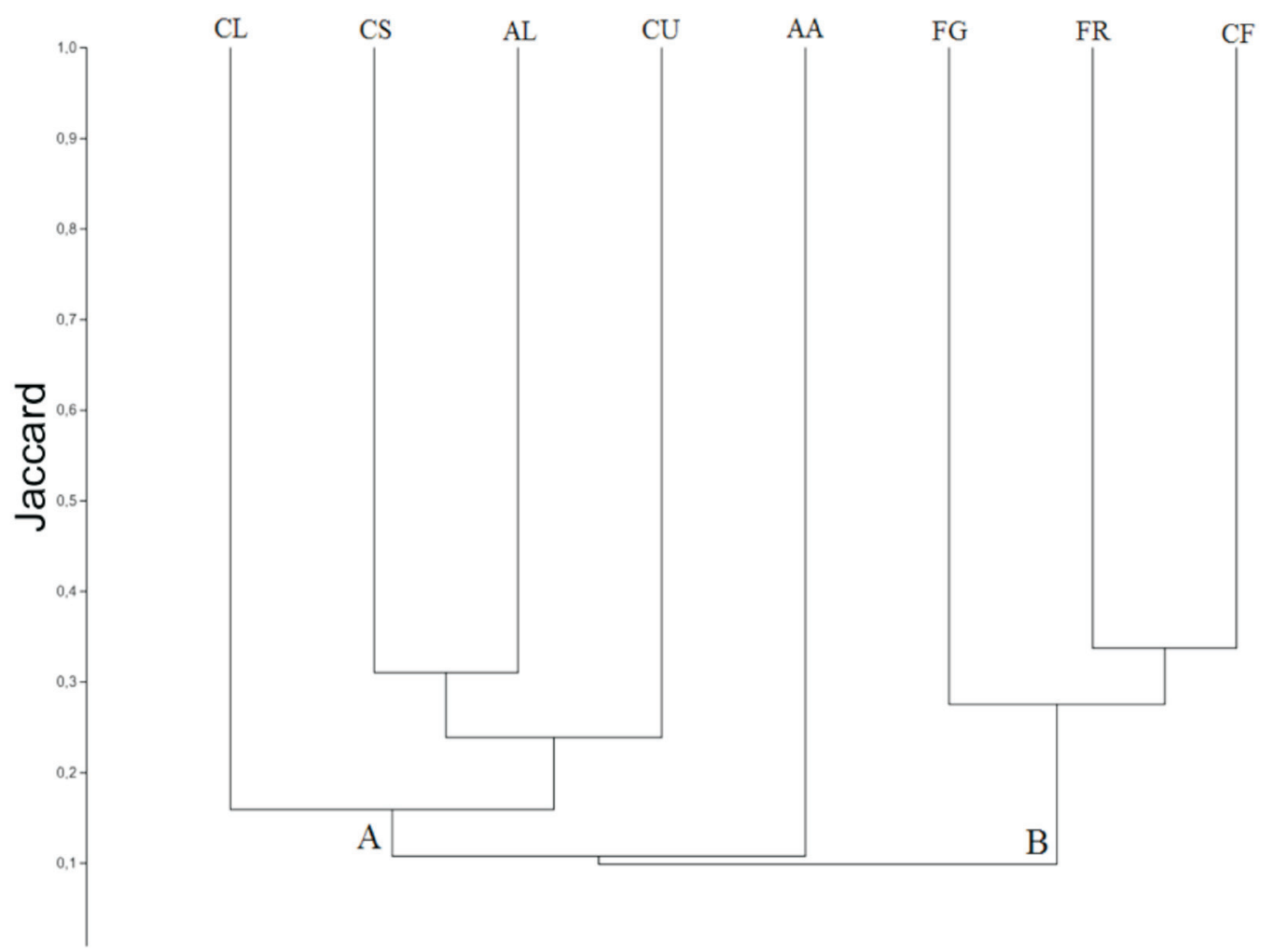

Figura 1. Dendrograma de agrupamento da análise do índice de similaridade de Jaccard entre os diferentes ambientes encontrados no Parque Estadual do Guartelá, PR, Brasil. CL: campo litólico; CS: campo seco; AL: ambiente ripário de lajeado; CU: campo úmido; AA: ambiente antropizado; FG: floresta de galeria; FR: floresta ripária do rio Iapó; CF: capão de floresta.

Figure 1. Cluster analysis of the Jaccard similarity index between the different environments found in Parque Estadual do Guartelá, Paraná State, Brazil. CL: litholic grasslands; CS: dry grasslands; AL: riparian slabstone ambient; CU: wet grasslands; AA: anthropic environment; FG: gallery forests; FR: Iapó riparian forest; CF: capon forest. 
perturbados, principalmente em relação à baixa densidade do sub-bosque. Em algumas áreas, o estrato herbáceo é desprovido de samambaias, fato pouco usual, considerando que diversas espécies (e.g. Ctenitis falciculada, Thelypteris retusa e Polystichum longecuspis Fée) são caracteristicamente encontradas em áreas perturbadas e em regeneração, usualmente em elevada densidade (Canestraro \& Kersten 2011). Nesses capões foram encontrados indivíduos de Ctenitis falciculata e Thelypteris retusa, entretanto esses eram raros e nunca formando grandes populações (a primeira encontrada em elevada densidade em outras formações florestais mais conservadas). Já Polystichum longecuspis não foi encontrada no PEG. Nos capões de floresta foram observadas 53 espécies, quatro delas exclusivas (Doryopteris majestosa, Leucotrichum schenckii, Rumohra adiantiformis e Thelypteris retusa).

Florestas de galeria: nesse ambiente foram encontradas 105 espécies (49 exclusivas), constituindo a formação com maior riqueza florística e número de espécies exclusivas. As espécies foram observadas principalmente ao longo de córregos, crescendo como rupícolas ou epífitas nas margens desses cursos d'água, ou ainda em barrancos úmidos no interior da floresta. Destaque para os gêneros Elaphoglossum e Phlegmariurus, por serem gêneros, no Estado do Paraná, mais comumente encontrados em áreas de Floresta Ombrófila Densa, especialmente em regiões montanhosas, e mais raramente em regiões planálticas no interior do Estado.

Floresta ripária do rio Iapó: nessa formação foram registradas 58 espécies (12 exclusivamente nela), crescendo principalmente na margem do rio Iapó, em áreas com alta incidência luminosa. Destaque para Thelypteris sp. nov. Além desta, Adiantum curvatum e Selaginella microphylla por serem espécies pouco coletadas no Estado, entretanto, no PEG, essas espécies são comumente encontradas nesse tipo de formação.

Ambientes ripários de lajeado: nesse ambiente foram observadas 25 espécies, três delas restritas

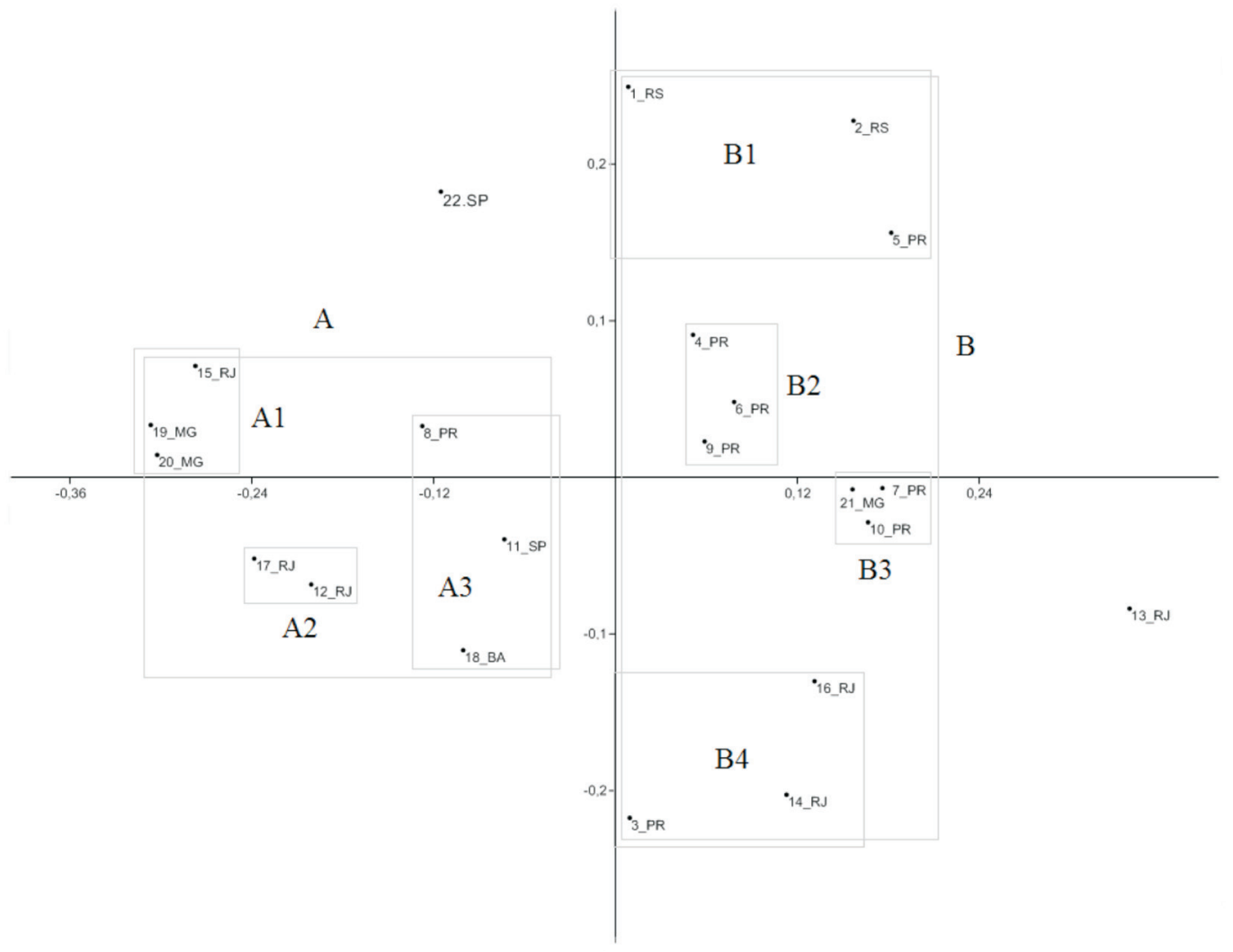

Figura 2. Análise de similaridade florística de 22 áreas de interesse (Floresta Atlântica Brasileira) com os respectivos números de referência listados na tabela 2.

Figure 2. Figure 2. Floristic similarity analysis of 22 areas of interest (Brazillian Atlantic Forest) with the respective reference numbers listed on table 2 .. 
(Pityrogramma calomelanos, Thelypteris opposita e Thelypteris pachyrhachis). Dentre as mais frequentes, destacam-se Blechnum austrobrasilianum, Blechnum polypodioides, Dicranopteris flexuosa, Lophosoria quadripinnata e Selaginella marginata, as quais usualmente formam grandes populações nos barrancos encontrados nas margens dos rios ou diretamente sobre as rochas do lajeado.

Campos secos: nessa formação foram verificadas 19 espécies, duas delas exclusivas (Anemia ferruginea e Phlegmariurus badinianus). Destacam-se as espécies Cyathea atrovirens e Cyathea corcovadensis, as quais constituem, visualmente, um dos principais componentes florísticos das formações campestres. Phlegmariurus badinianus é o primeiro registro da espécie no Estado do Paraná.

Campos úmidos: nos campos hidromórficos foram encontradas 17 espécies, duas exclusivas desse ambiente (Lycopodium clavatum e Palhinhaea camporum). Destacam-se as espécies dos gêneros Lycopodiella e Pseudolycopodiella por formarem grandes populações.

Campos litólicos: nos campos com afloramentos de arenito foram observadas 29 espécies, crescendo diretamente sobre os arenitos ou nos solos rasos na base e topo dos mesmos, cinco delas exclusivamente (Anemia sp. nov., Blechnum asplenioides, Cheilanthes goyazensis, Cyathea myriotricha e C. villosa).

Ambientes antropizados: em ambientes alterados foram verificadas nove espécies, três delas exóticas (Macrothelypteris torresiana, Pteris vittata e Thelypteris dentata) e duas espécies exclusivas (Pteris vittata e Thelypteris conspersa).

No dendrograma de agrupamento das formações do PEG (figura 1) é possível observar a reunião de dois grupos, o primeiro incluindo as formações herbáceo-arbustivas (A) e o segundo as formações florestais (B). No grupo A vê-se a segregação dos campos litólicos, explicada pela flora diferenciada encontrada nos afloramentos de arenito ou nos solos rasos no topo dos mesmos. Já a separação dos campos úmidos deve-se provavelmente à presença de solos hidricamente saturados e a seletividade imposta pelos mesmos, o que não é observado nas áreas de campo seco e ambiente de lajeado. Neste último, apesar da existir a influência direta de cursos d'água, o solo nunca se encontra saturado, pois a água é rapidamente drenada nos solos arenosos característicos desses ambientes.

No grupo B, representado pelas formações florestais, nota-se imediatamente a separação das florestas de galeria, causada pelos fatores abióticos únicos desse ambiente, como a elevada umidade do ar e a presença de pequenos riachos. Além disso, encontram-se abundantes afloramentos rochosos, responsáveis, assim como nas áreas de campo litólico, por notável diferenciação da flora em relação às outras formações semelhantes, evidenciando a importância dos afloramentos rochosos para a diversidade dos grupos estudados.

Nenhuma formação do PEG apresentou elevada similaridade florística em relação às outras, indicando que os oito ambientes considerados neste estudo apresentam a flora de samambaias e licófitas marcadamente distinta dos demais. Tal fato reforça ainda mais o papel da heterogeneidade ambiental na riqueza florística encontrada na área.

Das espécies encontradas (tabela 1), 52 são terrícolas, 23 epífitas, 20 rupícolas, duas lianas e 67 apresentam mais de uma forma de vida. Considerando todas as espécies epifíticas (epífitas obrigatórias, facultativas e acidentais), foram registradas 54 espécies.

O número de epífitas pode ser comparado ao encontrado em áreas de Floresta Ombrófila Densa (Labiak \& Prado 1998, Dittrich et al. 2005, Matos et al. 2010) e pode ser explicado pelas condições microclimáticas do PEG, especialmente nas florestas de galerias, onde a umidade relativa do ar é notavelmente mais elevada e constante, quando comparada com outras formações florestais do Parque, condições que providenciam ambientes propícios ao epifitismo (Kersten 2010). Desta forma, com os dados atualmente disponíveis, as samambaias e licófitas representam $42 \%$ das epífitas vasculares registradas no Parque.

A análise da distribuição geográfica (tabela 1) resultou em 13 espécies (8\%) com distribuição Circum-antártica, seis (3\%) na América e África, 57 (35\%) restritas ao Neotrópico, $46(28 \%)$ restritas à América do Sul, 41 (25\%) endêmicas do Brasil e duas (1\%) com distribuição incerta.

Em relação às espécies exóticas no continente americano, temos Deparia petersenii, Macrothelypteris torresiana, Pteris vittata e Thelypteris dentata. Essas espécies foram normalmente encontradas associadas à ambientes ruderais ou matas alteradas, e aparentemente não tem afetado seriamente a dinâmica da competição por habitats naturais. De qualquer forma, indivíduos isolados de Deparia petersenii, Macrothelypteris torresiana e Thelypteris dentata foram observados crescendo em ambientes de lajeado, florestas de galeria e na floresta ripária do rio Iapó, ambientes com baixa interferência antrópica resultantes do turismo local. 
Tabela 1. Lista das espécies de samambaias e licófitas do Parque Estadual do Guartelá, PR, Brasil. Distr.: Distribuição geográfica (NEO: neotropical; BR: Brasil; AFR: América e África; AMS: América do Sul; CA: Circum-Antártica). Formação (CS: campo seco; CF: capão de floresta; FG: floresta de galeria; FR: floresta ripária; CL: campo litólico; AL: ambiente ripário de lajeado; CU: campo úmido; AA: ambiente antropizado). Hábito (T: terrícola; R: rupícola; E: epífita; L: liana). Voucher (número de coletor de C. Michelon no herbário UPCB).

Table 1. Checklist of the ferns and lycophytes from the Parque Estadual do Guartelá, Paraná State, Brazil. Distr.: geographical distribution (NEO: neotropical; BR: Brazil; AFR: America and Africa; AMS: Southern America; CA: circum-Antarctic). Formation (CS: dry grasslands; CF: capon forest; FG: gallery forest; FR: riparian forest; CL: litholic grasslands; AL: riparian slabstone ambient; CU: wet grasslands; AA: anthropic environment). Habit (T: terrestrial; R: lithophyte; E: ephiphyte; L: climber). Voucher (collector number from C. Michelon at UPCB herbarium).

\begin{tabular}{|c|c|c|c|c|}
\hline $\begin{array}{l}\text { FAMÍLIA ( } n^{\circ} \text { de espécies) } \\
\text { Espécie }\end{array}$ & Distr. & Formação & Hábito & Voucher \\
\hline \multicolumn{5}{|l|}{ ANEMIACEAE (5) } \\
\hline Anemia ferruginea Kunth & NEO & CS & $\mathrm{T}$ & 1142 \\
\hline Anemia phyllitidis (L.) Sw. & NEO & CF, FG, FR & $\mathrm{T}, \mathrm{R}$ & 826 \\
\hline Anemia raddiana Link & $\mathrm{BR}$ & $\mathrm{CF}, \mathrm{FR}, \mathrm{CS}$ & $\mathrm{T}$ & 1069 \\
\hline Anemia tomentosa (Savigny) Sw. & NEO & FR, CS & $\mathrm{T}$ & 1033 \\
\hline Anemia sp. nov. & $?$ & $\mathrm{CL}$ & $\mathrm{R}$ & 942 \\
\hline \multicolumn{5}{|l|}{ ASPLENIACEAE (13) } \\
\hline Asplenium auritum $\mathrm{Sw}$. & AFR & FR & $\mathrm{R}$ & 879 \\
\hline Asplenium claussenii Hieron. & NEO & CF, FG, FR & $\mathrm{T}, \mathrm{E}, \mathrm{R}$ & 1124 \\
\hline Asplenium gastonis Fée & AMS & CF, FR & $\mathrm{T}, \mathrm{E}, \mathrm{R}$ & 922 \\
\hline Asplenium harpeodes Kunze & NEO & FG & $\mathrm{E}$ & 725 \\
\hline Asplenium inaequilaterale Willd. & $\mathrm{CA}$ & $\mathrm{CF}, \mathrm{FG}$ & $\mathrm{T}, \mathrm{R}$ & 1141 \\
\hline Asplenium incurvatum Fée & $\mathrm{BR}$ & FR & $\mathrm{R}$ & 867 \\
\hline Asplenium mucronatum C. Presl & AMS & FG & $\mathrm{E}$ & 1153 \\
\hline Asplenium oligophyllum Kaulf. & AMS & FG & E, R & 724 \\
\hline Asplenium pseudonitidum Raddi & $\mathrm{BR}$ & FG & $\mathrm{T}$ & 1026 \\
\hline Asplenium scandicinum Kaulf. & AMS & FG, FR & $\mathrm{E}$ & 1070 \\
\hline Asplenium serratum L. & NEO & FR & $\mathrm{T}$ & 998 \\
\hline Asplenium uniseriale Raddi & NEO & FG & $\mathrm{T}$ & 1108 \\
\hline $\begin{array}{l}\text { Hymenasplenium triquetrum (N. Murak. \& } \\
\text { R.C. Moran) L. Regalado \& Prada }\end{array}$ & AMS & FG & $\mathrm{R}$ & 1154 \\
\hline \multicolumn{5}{|l|}{ BLECHNACEAE (12) } \\
\hline Blechnum acutum (Desv.) Mett. & NEO & $\mathrm{CF}, \mathrm{FG}$ & $\mathrm{T}, \mathrm{E}, \mathrm{R}, \mathrm{L}$ & 929 \\
\hline Blechnum asplenioides Sw. & AMS & $\mathrm{CL}$ & $\mathrm{R}$ & 934 \\
\hline Blechnum austrobrasilianum de la Sota & AMS & CF, AL, FR & $\mathrm{T}$ & 814 \\
\hline Blechnum brasiliense Desv. & NEO & $\mathrm{CF}, \mathrm{AL}, \mathrm{FG}$ & $\mathrm{T}, \mathrm{R}$ & 1147 \\
\hline Blechnum $\times$ caudatum Cav. & NEO & FG, FR & $\mathrm{T}, \mathrm{R}$ & 1060 \\
\hline Blechnum cordatum (Desv.) Hieron. & AMS & $\begin{array}{c}\mathrm{CF}, \mathrm{AL}, \mathrm{FG}, \mathrm{FR}, \mathrm{CS}, \\
\mathrm{CU}\end{array}$ & $\mathrm{T}, \mathrm{R}$ & 789 \\
\hline Blechnum divergens (Kunze) Mett & NEO & FG & $\mathrm{R}$ & 1130 \\
\hline Blechnum gracile Kaulf. & NEO & FG, FR & $\mathrm{T}, \mathrm{R}$ & 773 \\
\hline Blechnum laevigatum Cav. & AMS & FG & $\mathrm{R}$ & 1028 \\
\hline Blechnum occidentale $\mathrm{L}$. & NEO & FR & $\mathrm{T}$ & 869 \\
\hline Blechnum polypodioides Raddi & NEO & FG, AL, FG, FR, CL & $\mathrm{T}, \mathrm{R}$ & 676 \\
\hline Blechnum schomburgkii (Klotzsch) C. Chr. & NEO & $\mathrm{AL}, \mathrm{CS}, \mathrm{CU}$ & $\mathrm{T}$ & 793 \\
\hline
\end{tabular}


Tabela 1 (continuação)

\begin{tabular}{|c|c|c|c|c|}
\hline $\begin{array}{l}\text { FAMÍLIA (nº de espécies) } \\
\text { Espécie }\end{array}$ & Distr. & Formação & Hábito & Voucher \\
\hline \multicolumn{5}{|l|}{ CYATHEACEAE (7) } \\
\hline Alsophila setosa Kaulf. & BR & \multirow{3}{*}{$\begin{array}{c}\text { CF, FG } \\
\text { CF, AL, FG, FR, CL, } \\
\text { CS, CU, AA } \\
\text { CF, FR, CS }\end{array}$} & $\mathrm{T}$ & 797 \\
\hline Cyathea atrovirens (Langsd. \& Fisch.) Domin & $\mathrm{BR}$ & & $\mathrm{T}, \mathrm{R}$ & 1051 \\
\hline Cyathea corcovadensis (Raddi) Domin & $\mathrm{BR}$ & & $\mathrm{T}$ & 1104 \\
\hline Cyathea delgadii Sternb. & NEO & $\mathrm{CF}, \mathrm{AL}, \mathrm{FG}, \mathrm{FR}$ & $\mathrm{T}, \mathrm{R}$ & 720 \\
\hline Cyathea myriotricha (Baker) R.C. Moran \& J. Prado & $\mathrm{BR}$ & $\mathrm{CL}$ & $\mathrm{R}$ & 859 \\
\hline Cyathea phalerata Mart. & $\mathrm{BR}$ & $\mathrm{CF}, \mathrm{FG}$ & $\mathrm{T}$ & 907 \\
\hline Cyathea villosa Humb. \& Bonpl. ex Willd. & AMS & $\mathrm{CL}$ & $\mathrm{T}$ & 1148 \\
\hline \multicolumn{5}{|l|}{ DENNSTAEDTIACEAE (3) } \\
\hline Dennstaedtia globulifera (Poir.) Hieron. & NEO & FG & $\mathrm{T}$ & 1106 \\
\hline Dennstaedtia obtusifolia (Willd.) T. Moore & NEO & \multirow{2}{*}{$\begin{array}{c}\mathrm{FG} \\
\mathrm{CF}, \mathrm{AL}, \mathrm{FR}, \mathrm{CS}, \mathrm{CU}, \\
\mathrm{AA}\end{array}$} & $\mathrm{T}$ & 1032 \\
\hline Pteridium arachnoideum (Kaulf.) Maxon & NEO & & $\mathrm{T}$ & 1285 \\
\hline \multicolumn{5}{|l|}{ DICKSONIACEAE (2) } \\
\hline Dicksonia sellowiana Hook. & NEO & $\mathrm{CF}, \mathrm{FG}$ & $\mathrm{T}$ & 1158 \\
\hline Lophosoria quadripinnata (J.F. Gmel.) C. Chr. & NEO & $\mathrm{CF}, \mathrm{AL}, \mathrm{FG}, \mathrm{CL}, \mathrm{CU}$ & $\mathrm{T}, \mathrm{R}$ & 786 \\
\hline \multicolumn{5}{|l|}{ DRYOPTERIDACEAE (18) } \\
\hline Arachniodes denticulata (Sw.) Ching & NEO & FG & $\mathrm{T}, \mathrm{E}, \mathrm{R}$ & 743 \\
\hline Ctenitis bigarellae Schwartsb. et al. & $\mathrm{BR}$ & FG & $\mathrm{T}$ & 1157 \\
\hline Ctenitis distans (Brack.) Ching & BR & $\mathrm{CF}, \mathrm{FG}$ & $\mathrm{T}$ & 1111 \\
\hline Ctenitis falciculata (Raddi) Ching & NEO & FG & $\mathrm{T}$ & 1122 \\
\hline Didymochlaena truncatula (Sw.) J.Sm. & $\mathrm{CA}$ & FG & $\mathrm{T}, \mathrm{R}$ & 743 \\
\hline Elaphoglossum brachyneuron (Fée) J.Sm. & NEO & FG & $\mathrm{R}$ & 740 \\
\hline Elaphoglossum burchellii (Baker) C. Chr. & NEO & $\mathrm{CF}, \mathrm{AL}, \mathrm{FG}, \mathrm{FR}$ & $\mathrm{T}, \mathrm{R}$ & 1068 \\
\hline Elaphoglossum lingua (C. Presl) Brack. & NEO & FR & $\mathrm{E}$ & 883 \\
\hline $\begin{array}{l}\text { Elaphoglossum macrophyllum (Mett. ex Kuhn) } \\
\text { H. Christ }\end{array}$ & $\mathrm{BR}$ & FG & $\mathrm{E}, \mathrm{T}$ & 1012 \\
\hline Elaphoglossum pachydermum (Fée) T.Moore & $\mathrm{BR}$ & $\mathrm{CF}, \mathrm{FG}, \mathrm{FR}, \mathrm{CL}$ & $\mathrm{T}, \mathrm{E}, \mathrm{R}$ & 1029 \\
\hline Elaphoglossum paulistanum Rosenst. & $\mathrm{BR}$ & FG & $\mathrm{E}$ & 1014 \\
\hline Elaphoglossum strictum (Raddi) T. Moore & $\mathrm{BR}$ & FG & $\mathrm{E}$ & 1000 \\
\hline Lastreopsis amplissima (C. Presl) Tindale & AMS & FG, FR & $\mathrm{T}, \mathrm{R}$ & 753 \\
\hline Lastreopsis effusa (Sw.) Tindale & NEO & FG & $\mathrm{T}$ & 886 \\
\hline $\begin{array}{l}\text { Megalastrum connexum (Kaulf.) A.R. Sm. \& } \\
\text { R.C. Moran }\end{array}$ & AMS & FG & $\mathrm{T}$ & 1074 \\
\hline $\begin{array}{l}\text { Mickelia scandens (Raddi) R.C. Moran, Sundue } \\
\text { \& Labiak }\end{array}$ & $\mathrm{BR}$ & FG & $\mathrm{L}$ & 729 \\
\hline Polybotrya cylindrica Kaulf. & $\mathrm{BR}$ & FG & $\mathrm{T}, \mathrm{L}$ & 1103 \\
\hline Rumohra adiantiformis (G. Forst.) Ching & $\mathrm{CA}$ & $\mathrm{CF}$ & $\mathrm{T}$ & 1048 \\
\hline \multicolumn{5}{|l|}{ GLEICHENIACEAE (3) } \\
\hline Dicranopteris flexuosa (Schrad.) Underw. & NEO & $\mathrm{AL}, \mathrm{CL}, \mathrm{CU}$ & $\mathrm{T}, \mathrm{R}$ & 820 \\
\hline Gleichenella pectinata (Willd.) Ching & NEO & $\mathrm{AL}, \mathrm{CU}$ & $\mathrm{T}$ & 1284 \\
\hline Sticherus lanuginosus (Fée) Nakai & NEO & $\mathrm{AL}, \mathrm{CL}, \mathrm{CU}$ & $\mathrm{T}, \mathrm{R}$ & 807 \\
\hline \multicolumn{5}{|l|}{ HYMENOPHYLLACEAE (16) } \\
\hline Abrodictyum rigidum (Sw.) Ebihara \& Dubuisson & $\mathrm{CA}$ & FG & $\mathrm{R}$ & 1121 \\
\hline
\end{tabular}


Tabela 1 (continuação)

\begin{tabular}{|c|c|c|c|c|}
\hline $\begin{array}{l}\text { FAMÍLIA ( } n^{\circ} \text { de espécies) } \\
\text { Espécie }\end{array}$ & Distr. & Formação & Hábito & Voucher \\
\hline $\begin{array}{l}\text { Didymoglossum hymenoides (Hedw.) Ebihara } \\
\text { \& Dubuisson }\end{array}$ & NEO & CF, FG, FR & $\mathrm{E}, \mathrm{R}$ & 937 \\
\hline Didymoglossum reptans (Sw.) Ebihara \& Dubuisson & NEO & FG & $\mathrm{E}, \mathrm{R}$ & 898 \\
\hline Hymenophyllum caudiculatum Mart & BR & FG & $\mathrm{R}$ & 732 \\
\hline Hymenophyllum elegans Spreng. & NEO & FG & $\mathrm{R}$ & 1042 \\
\hline Hymenophyllum fragile (Hedw.) C.V. Morton & NEO & FG & $\mathrm{R}$ & 911 \\
\hline Hymenophyllum hirsutum (L.) Sw. & NEO & FG & $\mathrm{R}$ & 909 \\
\hline Hymenophyllum polyanthos Sw. & $\mathrm{CA}$ & FG, FR & $\mathrm{E}, \mathrm{R}$ & 996 \\
\hline Hymenophyllum pulchellum Schltdl. \& Cham. & NEO & FG & $\mathrm{E}$ & 1099 \\
\hline $\begin{array}{l}\text { Polyphlebium angustatum (Carmich.) Ebihara } \\
\text { \& Dubuisson }\end{array}$ & NEO & FG & $\mathrm{E}, \mathrm{R}$ & 920 \\
\hline $\begin{array}{l}\text { Polyphlebium diaphanum (Kunth) Ebihara } \\
\text { \& Dubuisson }\end{array}$ & NEO & FG, FR & $\mathrm{R}$ & 1021 \\
\hline Polyphlebium pyxidiferum (L.) Ebihara \& Dubuisson & AFR & FG & E, R & 1010 \\
\hline Trichomanes anadromum Rosenst. & AMS & $\mathrm{CF}, \mathrm{FG}$ & $\mathrm{E}$ & 1050 \\
\hline Trichomanes pilosum Raddi & AMS & FG, CL & $\mathrm{R}$ & 690 \\
\hline Trichomanes polypodioides $\mathrm{L}$. & NEO & FG & E & 1097 \\
\hline Vandenboschia radicans (Sw.) Ebihara \& Dubuisson & $\mathrm{CA}$ & FG & $\mathrm{L}$ & 734 \\
\hline \multicolumn{5}{|l|}{ LINDSAEACEAE (4) } \\
\hline Lindsaea arcuata Kunze & NEO & $\mathrm{CF}, \mathrm{FG}$ & $\mathrm{T}$ & 721 \\
\hline Lindsaea botrychioides A. St.-Hil. & $\mathrm{BR}$ & $\mathrm{CF}, \mathrm{FG}$ & $\mathrm{T}$ & 897 \\
\hline Lindsaea quadrangularis Raddi & AMS & $\mathrm{CF}, \mathrm{FG}$ & $\mathrm{T}$ & 805 \\
\hline Lindsaea stricta (Sw.) Dryand. & NEO & $\mathrm{FR}, \mathrm{CL}$ & $\mathrm{T}, \mathrm{R}$ & 854 \\
\hline \multicolumn{5}{|l|}{ LYCOPODIACEAE (13) } \\
\hline Lycopodiella alopecuroides (L.) Cranfill & NEO & $\mathrm{CL}$ & $\mathrm{T}$ & 1094 \\
\hline Lycopodium clavatum $\mathrm{L}$. & AFR & $\mathrm{CU}$ & $\mathrm{T}$ & 790 \\
\hline Palhinhaea camporum B.Øllg. \& P.G. Windisch & AMS & $\mathrm{CU}$ & $\mathrm{T}$ & 694 \\
\hline Palhinhaea cernua (L.) Pic. Serm. & $\mathrm{CA}$ & $\mathrm{CU}$ & $\mathrm{T}$ & 1135 \\
\hline $\begin{array}{l}\text { Phlegmariurus badinianus (B. Øllg. \& P.G. Windisch) } \\
\text { B. Øllg. }\end{array}$ & $\mathrm{BR}$ & $\mathrm{CS}$ & $\mathrm{T}$ & 987 \\
\hline Phlegmariurus comans (Herter ex Nessel) B. Øllg. & BR & FG & $\mathrm{E}$ & 1091 \\
\hline Phlegmariurus flexibilis (Fée) B. Øllg. & BR & FG, FR & $\mathrm{E}$ & 1088 \\
\hline Phlegmariurus fontinaloides (Spring) B. Øllg. & $\mathrm{BR}$ & FG & $\mathrm{E}$ & 1089 \\
\hline Phlegmariurus heterocarpon (Fée) B. Øllg. & AMS & FG & $\mathrm{E}$ & 1004 \\
\hline Phlegmariurus mandiocanus (Raddi) B. Øllg. & AMS & FG, FR & $\mathrm{E}$ & 1092 \\
\hline Phlegmariurus quadrifariatus (Bory) B. Øllg. & $\mathrm{BR}$ & FR & $\mathrm{E}$ & 1156 \\
\hline Phlegmariurus reflexus (Lam.) B. Øllg.. & NEO & $\mathrm{AL}, \mathrm{CL}, \mathrm{CS}$ & $\mathrm{T}, \mathrm{R}$ & 969 \\
\hline \multicolumn{5}{|l|}{ MARATTIACEAE (1) } \\
\hline Danaea moritziana C. Presl & $\mathrm{BR}$ & FG & $\mathrm{T}, \mathrm{R}$ & 1102 \\
\hline \multicolumn{5}{|l|}{ OSMUNDACEAE (1) } \\
\hline Osmunda regalis $\mathrm{L}$. & AFR & $\mathrm{FR}, \mathrm{CU}$ & $\mathrm{T}$ & 806 \\
\hline \multicolumn{5}{|l|}{ POLYPODIACEAE (25) } \\
\hline Campyloneurum acrocarpon Fée & BR & $\mathrm{CF}, \mathrm{FG}$ & $\mathrm{E}$ & 913 \\
\hline
\end{tabular}


Tabela 1 (continuação)

\begin{tabular}{|c|c|c|c|c|}
\hline $\begin{array}{l}\text { FAMÍLIA ( } \mathrm{n}^{\circ} \text { de espécies) } \\
\text { Espécie }\end{array}$ & Distr. & Formação & Hábito & Voucher \\
\hline Campyloneurum austrobrasilianum (Alston) de la Sota & BR & CF, FR & $\mathrm{E}$ & 692 \\
\hline Campyloneurum minus Fée & AMS & FG & $\mathrm{E}, \mathrm{R}$ & 928 \\
\hline Campyloneurum nitidum (Kaulf.) C. Presl & AMS & $\mathrm{CF}, \mathrm{FG}, \mathrm{FR}$ & $\mathrm{E}, \mathrm{R}$ & 829 \\
\hline Cochlidium serrulatum (Sw.) L.E. Bishop & CA & $\mathrm{CF}, \mathrm{FG}, \mathrm{CL}$ & $\mathrm{E}, \mathrm{R}$ & 939 \\
\hline Leucotrichum schenckii (Hieron.) Labiak & BR & $\mathrm{CF}$ & $\mathrm{E}$ & 1153 \\
\hline Microgramma squamulosa (Kaulf.) de la Sota & MAS & $\mathrm{CF}, \mathrm{FR}$ & $\mathrm{E}, \mathrm{R}$ & 686 \\
\hline Microgramma vacciniifolia (Langsd. \& Fisch.) Copel. & NEO & FG, FR & $\mathrm{E}, \mathrm{R}$ & 880 \\
\hline Niphidium crassifolium (L.) Lellinger & NEO & $\mathrm{CF}, \mathrm{FG}$ & $E, R$ & 1086 \\
\hline Pecluma filicula (Kaulf.) M.G. Price & AMS & FR & $\mathrm{R}$ & 991 \\
\hline Pecluma paradiseae (Langsd. \& Fisch.) M.G. Price & BR & FR & $\mathrm{T}, \mathrm{R}$ & 995 \\
\hline Pecluma pectinatiformis (Lindm.) M.G. Price & AMS & $\mathrm{CF}, \mathrm{FG}$ & $\mathrm{E}, \mathrm{R}$ & 1133 \\
\hline Pecluma recurvata (Kaulf.) M.G. Price & AMS & CF, FG, FR & $\mathrm{E}, \mathrm{R}$ & 765 \\
\hline Pecluma sicca (Lindm.) M.G. Price & AMS & CF, FR & $\mathrm{E}, \mathrm{R}$ & 1062 \\
\hline Pecluma singeri (de la Sota) M.G. Price & AMS & FG & $\mathrm{E}$ & 890 \\
\hline Pecluma truncorum (Lindm.) M.G. Price & AMS & FG & $\mathrm{E}$ & 1095 \\
\hline $\begin{array}{l}\text { Phlebodium areolatum (Humb. \& Bonpl. ex Willd.) } \\
\text { J. Sm. }\end{array}$ & NEO & $\mathrm{CF}, \mathrm{FR}$ & $\mathrm{E}, \mathrm{R}$ & 1066 \\
\hline Pleopeltis hirsutissima (Raddi) de la Sota & AMS & $\mathrm{CF}, \mathrm{FG}, \mathrm{FR}, \mathrm{CL}$ & $\mathrm{T}, \mathrm{E}, \mathrm{R}$ & 687 \\
\hline Pleopeltis macrocarpa (Bory ex Willd.) Kaulf. & AFR & FR & $\mathrm{E}, \mathrm{R}$ & 1112 \\
\hline Pleopeltis pleopeltifolia (Raddi) Alston & AMS & CF, FG, FR & $\mathrm{E}, \mathrm{R}$ & 716 \\
\hline Pleopeltis squalida (Vell.) de la Sota & AMS & CF, FR & $\mathrm{E}$ & 912 \\
\hline Serpocaulon catharinae (Langsd. \& Fisch.) A.R. Sm. & AMS & $\mathrm{CF}, \mathrm{FG}, \mathrm{FR}, \mathrm{CL}$ & $\mathrm{T}, \mathrm{E}, \mathrm{R}$ & 776 \\
\hline Serpocaulon latipes (Langsd. \& Fisch.) A.R. Sm. & $\mathrm{BR}$ & $\mathrm{AL}, \mathrm{FR}, \mathrm{CL}, \mathrm{CS}$ & $\mathrm{T}, \mathrm{R}$ & 1067 \\
\hline $\begin{array}{l}\text { Serpocaulon meniscifolium (Langsd. \& Fisch.) } \\
\text { A.R. Sm. }\end{array}$ & $\mathrm{BR}$ & $\mathrm{CF}, \mathrm{FG}, \mathrm{FR}, \mathrm{CL}$ & $\mathrm{T}, \mathrm{E}, \mathrm{R}$ & 889 \\
\hline Serpocaulon vacillans (Link) A.R. Sm. & AMS & $\mathrm{CF}, \mathrm{FG}, \mathrm{CL}$ & $\mathrm{T}, \mathrm{R}$ & 795 \\
\hline \multicolumn{5}{|l|}{ PTERIDACEAE (22) } \\
\hline Adiantopsis chlorophylla (Sw.) Fée & AMS & $\mathrm{CF}, \mathrm{CS}, \mathrm{CU}, \mathrm{AA}$ & $\mathrm{T}$ & 1034 \\
\hline Adiantopsis radiata $(\mathrm{L}$.) Fée & NEO & CF, FG, FR & $\mathrm{T}$ & 728 \\
\hline Adiantum curvatum Kaulf. & BR & FG, FR & $\mathrm{T}$ & 1140 \\
\hline Adiantum pseudotinctum Hieron. & AMS & FR, AA & $\mathrm{T}, \mathrm{R}$ & 852 \\
\hline Adiantum raddianum C. Presl & NEO & FG, CL & $\mathrm{T}, \mathrm{R}$ & 942 \\
\hline Cheilanthes goyazensis (Taub.) Domin & $\mathrm{BR}$ & $\mathrm{CL}$ & $\mathrm{R}$ & 856 \\
\hline Doryopteris crenulans (Fée) H. Christ & AMS & $\mathrm{CL}, \mathrm{CS}, \mathrm{CU}$ & $\mathrm{T}, \mathrm{R}$ & 712 \\
\hline Doryopteris lomariacea (Kunze) Klotzsch & AMS & $\mathrm{CL}, \mathrm{CS}, \mathrm{CU}$ & $\mathrm{T}, \mathrm{R}$ & 675 \\
\hline Doryopteris majestosa J.C. Yesilyurt & AMS & $\mathrm{CF}$ & $\mathrm{T}$ & 1120 \\
\hline Doryopteris ornithopus (Mett.) J. Sm. & BR & $\mathrm{CL}, \mathrm{CS}$ & $\mathrm{T}, \mathrm{R}$ & 684 \\
\hline Doryopteris pentagona Pic. Serm. & AMS & $\mathrm{CF}, \mathrm{FG}, \mathrm{FR}, \mathrm{AA}$ & $\mathrm{T}, \mathrm{R}$ & 824 \\
\hline $\begin{array}{l}\text { Jamesonia areniticola (Schwartsb. \& Labiak) } \\
\text { Christenh. }\end{array}$ & $\mathrm{BR}$ & $\mathrm{FR}, \mathrm{CL}$ & $\mathrm{R}$ & 904 \\
\hline Jamesonia myriophylla (Sw.) Christenh. & $\mathrm{BR}$ & $\mathrm{CL}, \mathrm{CU}$ & $\mathrm{T}, \mathrm{R}$ & 785 \\
\hline Pityrogramma calomelanos (L.) Link & CA & $\mathrm{AL}$ & $\mathrm{T}$ & 668 \\
\hline Polytaenium lineatum (Sw.) J. Sm. & NEO & FG & $\mathrm{E}$ & 1002 \\
\hline
\end{tabular}


Tabela 1 (continuação)

\begin{tabular}{|c|c|c|c|c|}
\hline $\begin{array}{l}\text { FAMÍLIA ( }{ }^{\circ} \text { de espécies) } \\
\text { Espécie }\end{array}$ & Distr. & Formação & Hábito & Voucher \\
\hline Pteris decurrens $\mathrm{C}$. Presl & AMS & FG & $\mathrm{T}$ & 1008 \\
\hline Pteris deflexa Link & NEO & FG & $\mathrm{T}$ & 1113 \\
\hline Pteris lechleri Mett. & NEO & FG & $\mathrm{T}$ & 1160 \\
\hline Pteris splendens Kaulf. & AMS & FG & $\mathrm{T}, \mathrm{E}, \mathrm{R}$ & 755 \\
\hline Pteris vittata $\mathrm{L}$. & $\mathrm{CA}$ & $\mathrm{AA}$ & $\mathrm{T}, \mathrm{R}$ & 1049 \\
\hline Vittaria graminifolia Kaulf. & NEO & FG & $\mathrm{E}$ & 908 \\
\hline Vittaria lineata (L.) Sm. & NEO & $\mathrm{CF}, \mathrm{FG}, \mathrm{FR}$ & $\mathrm{E}, \mathrm{R}$ & 1064 \\
\hline \multicolumn{5}{|l|}{ SELAGINELLACEAE (3) } \\
\hline Selaginella flexuosa Spring & BR & FG, FR, CL & $\mathrm{E}, \mathrm{R}$ & 1031 \\
\hline $\begin{array}{l}\text { Selaginella marginata (Humb. \& Bonpl. ex Willd.) } \\
\text { Spring }\end{array}$ & NEO & $\mathrm{AL}, \mathrm{FG}, \mathrm{FR}, \mathrm{CU}$ & $\mathrm{T}$ & 811 \\
\hline Selaginella microphylla (Kunth) Spring & MAS & FR & $\mathrm{E}, \mathrm{R}$ & 868 \\
\hline \multicolumn{5}{|l|}{ THELYPTERIDACEAE (14) } \\
\hline Macrothelypteris torresiana (Gaudich.) Ching & $\mathrm{CA}$ & $\mathrm{AL}, \mathrm{FR}, \mathrm{AA}$ & $\mathrm{T}, \mathrm{R}$ & 662 \\
\hline Thelypteris amambayensis Ponce & AMS & $\mathrm{CF}, \mathrm{AL}, \mathrm{FG}$ & $\mathrm{T}, \mathrm{R}$ & 663 \\
\hline Thelypteris araucariensis Ponce & $\mathrm{BR}$ & CF, FG, FR & $\mathrm{T}$ & 752 \\
\hline Thelypteris conspersa (Schrad.) A.R. Sm. & NEO & AA & $\mathrm{T}$ & 667 \\
\hline Thelypteris dentata (Forssk.) E.P. St. John & $\mathrm{CA}$ & $\mathrm{CF}, \mathrm{AL}, \mathrm{FR}, \mathrm{CS}, \mathrm{AA}$ & $\mathrm{T}$ & 767 \\
\hline Thelypteris hatschbachii A.R. Sm. & $\mathrm{BR}$ & FR & $\mathrm{T}$ & 865 \\
\hline Thelypteris opposita (Vahl) Ching & NEO & $\mathrm{AL}$ & $\mathrm{T}$ & 660 \\
\hline Thelypteris pachyrhachis (Kunze ex Mett.) Ching & AMS & $\mathrm{AL}$ & $\mathrm{T}$ & 644 \\
\hline Thelypteris ptarmica (Kunze ex Mett.) C.F. Reed & $\mathrm{BR}$ & FG, FR & $\mathrm{R}$ & 739 \\
\hline Thelypteris raddi (Rosenst.) Ponce & $\mathrm{BR}$ & FG & $\mathrm{T}$ & 1127 \\
\hline Thelypteris retusa (Sw.) Reed & $\mathrm{BR}$ & $\mathrm{CF}$ & $\mathrm{T}$ & 802 \\
\hline Thelypteris rivularioides (Fée) Abbiatti & AMS & $\mathrm{AL}, \mathrm{CS}, \mathrm{CU}$ & $\mathrm{T}$ & 924 \\
\hline Thelypteris scabra (C. Presl) Lellinger & AMS & FG & $\mathrm{T}, \mathrm{R}$ & 932 \\
\hline Thelypteris sp. nov. & $?$ & FR & $\mathrm{T}, \mathrm{R}$ & 999 \\
\hline \multicolumn{5}{|l|}{ WOODSIACEAE (2) } \\
\hline Deparia petersenii (Kunze) M. Kato & $\mathrm{CA}$ & $\mathrm{AL}, \mathrm{FG}$ & $\mathrm{T}, \mathrm{R}$ & 989 \\
\hline Diplazium cristatum (Desr.) Alston & NEO & FG & $\mathrm{T}$ & 756 \\
\hline
\end{tabular}

$\mathrm{Na}$ análise de similaridade florística de 22 áreas na Floresta Atlântica Brasileira (figura 2, tabela 2) nota-se a presença de diversos grupos bem definidos, arranjados, principalmente, de acordo com o tipo de vegetação dominante e a proximidade geográfica entre as áreas. Existem, de qualquer forma, algumas exceções discutidas mais adiante.

Primeiramente é possível notar a formação de dois grandes grupos. O primeiro (Grupo A) é composto principalmente por áreas litorâneas das regiões Sudeste e Nordeste, cobertas por Florestas Estacionais Semideciduais e Florestas Ombrófilas Densas de Terras Baixas e Sub-Montanas. O segundo (Grupo B) é formado por áreas interioranas ou serranas das regiões Sul e Sudeste, cobertas por diversos tipos de vegetação, como Florestas Ombrófilas Mistas (FOM) e Densas (FOD), Florestas Estacionais Semideciduais (FES) e Campos (CAM).

Dentro do Grupo A, verifica-se a formação de três subgrupos (A1, A2 e A3). Os subgrupos A1 e A2 unem-se pela proximidade geográfica (região Sudeste) e pelo tipo de vegetação predominante (FOD e FES). De certa maneira, a formação deste grupo concorda com os dados de Oliveira Filho \& Fontes (2000) de que as Florestas Ombrófilas Atlânticas e as Florestas Estacionais Semideciduais do Sudeste brasileiro constituem um contínuo de distribuição de espécies. Ainda segundo estes autores, a flora arbórea da FES 
seria um subconjunto das Florestas Ombrófilas Mista e Densa, onde se desenvolve uma seleção de espécies capazes de suportar uma estação seca prolongada. O subgrupo A3 apresenta-se unido apesar da distância geográfica significativa entre as áreas (regiões Sul, Sudeste e Nordeste). Esse agrupamento reflete as peculiaridades de dispersão das samambaias e licófitas, as quais apresentam os padrões florísticos mais fortemente associados às condições ambientais e disponibilidade de habitats do que à distância geográfica entre as localidades (Moran 2008).

Dentro do Grupo B é possível notar a reunião de quatro subgrupos distintos (B1, B2, B3 e B4). O subgrupo B1 reflete o componente sulino de Mata Atlântica, constituído inteiramente por áreas de Florestas Ombrófilas Mistas interioranas, mais afastadas do Oceano Atlântico, nos Estados do Paraná e Rio Grande do Sul. O subgrupo B2 é fortemente influenciado tanto pela proximidade física quanto pelas semelhanças ambientais, incluindo a área deste estudo. Localizadas nos Campos Gerais do Estado do Paraná, essas três áreas (PEG, Parque Estadual de Vila Velha e Parque Ecológico da Klabin) apresentam uma heterogeneidade ambiental elevada, característica marcante da região. Além dessa ampla variação ambiental, esse grupo reúne todas as áreas que apresentam como vegetação predominante os Campos. O subgrupo B3 reúne áreas de tensão ecológica (ecótono entre FOM e FOD) nas regiões Sul e Sudeste, onde prevalecem os componentes montanos com marcante influência do componente alto-montano. O subgrupo B4 é formado por áreas semelhantes ao subgrupo anterior, tanto em relação à distância geográfica e tipos vegetacionais, entretanto, esse subgrupo é formado inteiramente por áreas de FOD montana com influência do componente alto-montano.

As áreas 13 (Parque Nacional do Itatiaia) e 22 (Serra do Cuscuzeiro) aparecem distantes de todas as outras áreas e distantes entre si, indicando uma baixa similaridade florística com as áreas restantes. A diferenciação da primeira é esperada, uma vez que Condack (2006) considerou apenas o componente alto-montado do Parque. Já a segunda, provavelmente deve-se pela expressão mais significativa do Cerrado (sensu lato), uma formação ausente ou pouco expressiva nas outras áreas selecionadas para esta análise.

Apesar do foco central deste estudo não ser uma análise biogeográfica detalhada, o resultado desta breve investigação demonstra que estudos florísticos são imprescindíveis para o desenvolvimento de padrões de distribuição e biodiversidade, além de funcionarem como fontes de informação para ações conservacionistas (Funk \& Richardson 2002, Funk 2006).

É importante ressaltar a elevada riqueza de Hymenophyllaceae (16 espécies) e de Phlegmariurus (oito espécies). No Estado do Paraná, são grupos mais comumente encontrados em áreas de Floresta Ombrófila Densa, como as regiões montanhosas da Serra do Mar, e raramente nas regiões planálticas do interior do Estado.

Também vale a menção do gênero Blechnum, com 12 espécies. Além destas, alguns materiais coletados permanecem sem confirmação específica, tratando-se, possivelmente, de híbridos entre as espécies de frondes monomorfas encontradas na área. Esse processo de hibridação é frequente e bem documentado dentro do grupo, sendo que esses híbridos podem se tornar abundantes pela reprodução por estolões (Moran 1995). De qualquer forma, estudos adicionais são necessários para confirmar a origem híbrida desses espécimes, e, portanto, optou-se por não inclui-los na listagem de espécies.

Arachniodes denticulada, Elaphoglossum brachyneuron, E. strictum, Leucotrichum schenckii, Phlegmariurus comans, $P$. flexibilis, $P$. heterocarpon e P. quadrifariatus constituem o primeiro registro no Segundo Planalto Paranaense.

Em relação às espécies com grande interesse para a conservação no Estado, destacam-se:

1) Anemia ferruginea: espécie rara no Paraná, sendo conhecidas apenas duas populações, uma no PEG e outra no Parque Estadual do Cerrado;

2) Anemia sp. nov.: trata-se de uma espécie nova, atualmente sendo descrita por Mickel (dados não publicados);

3) Cheilanthes goyazensis: é o primeiro registro da espécie no Estado;

4) Ctenitis bigarellae: espécie endêmica da região dos campos gerais no Estado do Paraná. Esta é a segunda localidade onde o táxon foi encontrado;

5) Cyathea myriotricha: espécie rara e característica por crescer em fendas e frestas de paredões rochosos. Endêmica dos Estados do Paraná e Minas Gerais;

6) Cyathea villosa: apesar de ser uma espécie amplamente distribuída na América do Sul, parece ser rara noEstado do Paraná, devido aos poucos registros encontrados; 
Tabela 2. Relação das áreas utilizadas na análise florística. FED: Floresta Estacional Decidual, FOM: Floresta Ombrófila Mista, CAM: Campos, FOD: Floresta Ombrófila Densa, FES: Floresta Estacional Semidecidual, CER: Cerrado, CA: Campo de Altitude, CR: Complexo Rupestre.

Table 2. Listing of the areas used in the floristic analysis. FED: Deciduous Forest, FOM: Mixed Ombrophilous Forest, CAM: Grasslands, FOD: Dense Ombrophilous Forest, FES: Semideciduous Forest, CER: Savannah, CA: Montane Grasslands, CR: Rupestrial Complex.

\begin{tabular}{llcrl}
\hline Número. Nome da área - Estado Tipo de vegetação & Altitude $(\mathrm{m})$ & $\mathrm{N}^{o}$ de spp. & Fonte \\
\hline 1. Morro da Harmonia - RS & FED, FOM & $250-700$ & 71 & Steffens \& Windisch (2007) \\
2. FLONA Canela - RS & CAM, FOM & 770 & 58 & Schmitt et al. (2006) \\
3. P.E. Pico do Marumbi - PR & FOD & 630 & 81 & Dittrich et al. (2005) \\
4. PEc da Klabin - PR & CAM, FOM, FES & 885 & 121 & Sakagami (2006) \\
5. P.M. do Barigui - PR & FOM & 900 & 64 & Dittrich et al. (1999) e Kozera et al. \\
6. P.E. de Vila Velha - PR & CAM, FOM & $800-1.100$ & 152 & Schwartsburd \& Labiak (2007) \\
7. Mananciais da Serra - PR & FOM, FOD & 1.030 & 81 & Michelon \& Kersten (dados não \\
8. Ilha do Mel - PR & FOD & $0-150$ & 114 & Salino et al. (2005) \\
9. P.E. do Guartelá - PR & CAM, CER, FOM & $780-1.100$ & 164 & Presente estudo \\
10. Morro do Canal - PR & FOM, FOD & $950-1.370$ & 115 & Michelon (dados não publicados) \\
11. P.E. Jacupiranga - SP & FOD & $10-1.310$ & 212 & Salino \& Almeida (2008) \\
12. RPPN Rio das Pedras - RJ & FOD & $20-1.050$ & 117 & Mynssen \& Windisch (2004) \\
13. P.N. do Itatiaia - RJ & FOD, CA & $1.800-2.790$ & 135 & Condack (2006) \\
14. R.E. Macaé de Cima - RJ & FOD & $880-1.700$ & 81 & Sylvestre (1997) \\
15. REBIO Poço das Antas - RJ FOD & $20-60$ & 62 & Reis (1998) \\
16. P.N. Serra dos Órgãos - RJ & FOD & $1.100-1.600$ & 116 & Engelmann et al. (2007) \\
17. E.E. do Paraíso - RJ & FOD & $20-1.350$ & 102 & Jascone \& Miguel (2007) \\
18. RPPN Serra Bonita - BA & FOD & $300-1.080$ & 182 & Matos et al. (2010) \\
19. P.E. do Rio Doce - MG & FES & $230-510$ & 123 & Melo \& Salino (2002) \\
20. E.B. de Caratinga - MG & FES & $400-680$ & 102 & Melo \& Salino (2002) \\
21. APA Fernão Dias - MG & FES, FOD, FOM & $1.000-2.070$ & 173 & Melo \& Salino (2007) \\
22. Serra do Cuscuzeiro - SP & FES, CER & $800-1.050$ & 113 & Salino (1996) \\
\hline
\end{tabular}

7) Dicksonia sellowiana: espécie constante na lista da flora ameaçada de extinção do Brasil;

8) Jamesonia areniticola: espécie endêmica dos afloramentos de arenito nos Estados do Paraná e São Paulo;

9) Lindsaea stricta: apesar de ser uma espécie amplamente distribuída na América Tropical, e também no Brasil, parece ser uma espécie rara no Estado do Paraná, sendo este o segundo registro para o Estado;

10) Phlegmariurus badinianus: é o primeiro registro da espécie no Estado do Paraná;

11) Thelypteris araucariensis: espécie aparentemente endêmica da Floresta com Araucária;

12) Thelypteris sp. nov.: trata-se de uma espécie nova em processo de descrição.

\section{Agradecimentos}

Ao Programa de Pós-Graduação em Botânica da UFPR; ao CNPq, pela concessão da bolsa de Mestrado ao primeiro Autor. Ao Dr. Rodrigo de Andrade Kersten, pelo auxílio na descrição das formações do PEG; e aos revisores, pelas sugestões e correções.

\section{Literatura citada}

Canestraro, B.K. \& Kersten, R.A. 2011. A comunidade de pteridófitas terrícolas em diferentes estágios sucessionais de uma Floresta Ombrófila Mista, Fazenda Rio Grande, Paraná, Brasil. Monografia de Conclusão de Curso, Pontifícia Universidade Católica do Paraná, Curitiba. 
Carmo, M.R.B. 2006. Caracterização fitofisionômica do Parque Estadual do Guartelá, Município de Tibagi, Estado do Paraná. Tese de Doutorado, Universidade Estadual Paulista "Júlio de Mesquita Filho", Rio Claro.

Christenhusz, M.J.M., Schwartsbrud, P.B. \& Labiak, P.H. 2009. Hymenophyllum filmenofilicum (Hymenophyllaceae, Pteridophyta): a new epipetric filmy fern from Paraná, southern Brazil. Kew Bulletin 64: 175-178.

Christenhusz, M.J.M., Xian-Chun, Z. \& Schneider, H. 2011. A linear sequence of extant families and genera of lycophytes and ferns. Phytotaxa 19: 7-54.

Condack, J.P.S. 2006. Pteridófitas ocorrentes na região alto Montana do Parque Nacional do Itatiaia: análise florística e estrutural. Tese de Doutorado, Instituto de Pesquisas Jardim Botânico do Rio de Janeiro, Rio de Janeiro.

Diedrichs, L.A. 1995. O processo de criação do Parque Estadual do Guartelá. Monografia de Especialização, Universidade Federal do Paraná, Curitiba.

Dittrich, V.A.O., Kozera, C. \& Menezes-Silva, S. 1999. Levantamento florístico dos epífitos vasculares do Parque Barigüi, Curitiba, Paraná, Brasil. Iheringia 52: 11-21.

Dittrich, V.A.O., Waechter, J.L. \& Salino, A. 2005. Species richness of pteridophytes in a montane Atlantic rain forest plot of Southern Brazil. Acta Botanica Brasilica 19: 519-525.

Engelmann, R.A., Wesenberg, J. \& Morawetz, W. 2007. Pteridófitas e begoniáceas no sub-bosque da Mata Atlântica na parte oriental do Parque Nacional da Serra dos Órgãos, Teresópolis, RJ, Brasil. In: C. Cronemberger \& E.B. Viveiros-de-Castro (eds.). Ciência e Conservação na Serra dos Órgãos. IBAMA. Brasília, pp. 83-104.

Forzza, R.C., Leitman, P.M., Costa, A.F., Carvalho Jr., A.A., Peixoto, A.L., Walter, B.M.T., Bicudo, C., Zappi, D., Costa, D.P., Lleras, E., Martinelli, G., Lima, H.C., Prado, J., Stehmann, J.R., Baumgratz, J.F.A., Pirani, J.R., Sylvestre, L., Maia, L.C., Lohmann, L.G., Queiroz, L.P., Silveira, M., Coelho, M.N., Mamede, M.C., Bastos, M.N.C., Morim, M.P., Barbosa, M.R., Menezes, M., Hopkins, M., Secco, R., Cavalcanti, T.B. \& Souza, V.C. 2010. Catálogo de Plantas e Fungos do Brasil. Instituto de Pesquisas Jardim Botânico do Rio de Janeiro / Andréa Jakobsson Estúdio, Rio de Janeiro.

Funk, V.A. 2006. Floras: a model for biodiversity studies or a thing of the past? Taxon 55: 581-588.

Funk, V.A. \& Richardson, K.S. 2002. Systematic data in biodiversity studies: use it or lose it. Systematic Biology 51: 303-316.

Hammes, Ø., Harper, D.A.T. \& Ryan, P.D. 2001. PAST: Paleontological Statistics Software Package for Eduacation and Data Analysis. Palaeontologia Electronica 4: 1-9.
Hornes, K.L., Guimarães, G.B. \& Palhares, J.M. 2008. Feições geomorfológicas da Reserva Particular do Patrimônio Natural (RPPN) Itaytyba - Tibagi, Paraná. Plêiade 2: 17-27.

IAP. 2002. Plano de Manejo do Parque Estadual do Guartelá. Instituto Ambiental do Paraná, Curitiba. http:// www.iap.pr.gov.br (acesso em 03.08.2010).

Jascone, C.E.S. \& Miguel, J.R. 2007. Pteridoflora da Estação Ecológica Estadual do Paraíso, Rio de Janeiro, Brasil - Resultados Preliminares. Saúde \& Ambiente em Revista 2: 37-43.

Kersten, R.A. 2010. Epífitas vasculares - Histórico, participação taxonômica e aspectos relevantes, com ênfase na Mata Atlântica. Hoehnea 37: 9-38.

Klein, R.M. \& Hatschbach, G. 1971. Fitofisionomia e notas complementares sobre o mapa fitogeográfico de Quero-Quero (Paraná). Boletim Paranaense de Geociências 28-29: 159-188.

Kozera, C., Dittrich, V.A.O. \& Silva, S.M. 2006. Composição florística da Floresta Ombrófila Mista Montana do Parque Municipal do Barugüi, Curitiba, PR. Floresta 36: 45-58.

Labiak, P.H. \& Prado, J. 1998. Pteridófitas epífitas da Reserva Volta Velha, Itapoá - Santa Catarina, Brasil. Boletim do Instituto de Botânica 11: 1-79.

Maack, R. 1981. Geografia física do estado do Paraná, 2 ed. José Olympio, Rio de Janeiro.

Matos, F.B., Amorin, A.M. \& Labiak, P.H. 2010. The ferns and lycophytes of a montane tropical forest in southern Bahia, Brazil. Journal of Botanical Research Institute 4: 333-346.

Melo, M.S. 2000. Canyon Guartelá. In: C. Schobbenhaus, D.A. Campos, E.T. Queiroz, M. Winge \& M. BerbertBorn (eds.). Sítios geológicos e paleontológicos do Brasil. http://www.unb.br/ig/sigeb/sitio094/sitio094. htm (acesso em 13.06.2010).

Melo, L.C.N. \& Salino, A. 2002. Pteridófitas de duas áreas de floresta da bacia do Rio Doce no Estado de Minas Gerais, Brasil. Lundiana 3: 129-139.

Melo, L.C.N. \& Almeida, T.E. 2007. Pteridófitas em fragmentos florestais da APA Fernão Dias, Minas Gerais. Rodriguésia 58: 207-220.

Melo, A.S. \& Hepp, L.U. 2008. Ferramentas estatísticas para análises de dados provenientes de biomonitoramento. Oecologia Brasiliensis 12: 463-486.

Minchin, P.R. 1987. An evaluation of the relative robustness of techniques for ecological ordination. Vegetatio 69: 89-107.

Moran, R.C. 1995. Blechnaceae. In: G. Davidse, M. Sousa \& S. Knapp (eds). Flora Mesoamericana. V. 1: Psilotaceae a Salviniaceae. Ciudad de México: Universidad Nacional Autónoma de México. pp. 325333. 
Moran, R.C. 2008. Diversity, biogeography and floristics. In: T.A. Ranker \& C.H. Haufler (eds.). Biology and Evolution of Ferns and Lycophytes. Cambridge University Press, Cambridge, pp. 367-394.

Mynssen, C.M. \& Windisch, P.G. 2004. Pteridófitas da Reserva Rio Das Pedras, Mangaratiba, RJ, Brasil. Rodriguésia 55: 125-156.

Oliveira-Filho, A.T. \& Fontes, M.A.L. 2000. Patterns of floristic differentiation among Atlantic Forests in Southeastern Brazil and the influence of climate. Biotropica 32: 793-810.

Past. 2001. Paleontological Statistics. http:/folk.uio.no/ ohammer/past/ (acesso em 02.12.2011).

Reis, C.A. 1998. Pteridófitas da Reserva Biológica de Poço das Antas, Município de Silva Jardim - Rio de Janeiro: Lista das espécies e Análise florística. Monografia de Graduação, Universidade Federal Rural do Rio de Janeiro, Rio de Janeiro.

Retzlaf, J.G., Stipp, N.A.F. \& Archela, E. 2006. Breve síntese geológica e geomorfológica da área do Parque Estadual do Guartelá no Estado do Paraná. Geografia 15: 95-106.

Sakagami, C.R. 2006. Pteridófitas do Parque Ecológico da Klabin, Telêmaco Borba, Paraná, Brasil. Dissertação de Mestrado, Universidade Federal do Paraná, Curitiba.

Salino, A. 1996. Levantamento das Pteridófitas da Serra do Cuscuzeiro, Analândia, SP, Brasil. Revista Brasileira de Botânica 19: 173-178.

Salino, A. \& Almeida, T.E. 2008. Pteridófitas do Parque Estadual do Jacupiranga, SP, Brasil. Acta Botanica Brasilica 22: 983-991.

Salino, A., Silva, S.M., Dittrich, V.A.O. \& Britez, R.M. 2005. Flora pteridofítica. In: M.C.N. Marques \& R.M. Britez (eds.). História natural e conservação da Ilha do Mel. Editora Universidade Federal do Paraná, Curitiba, pp. 85-101.

Schwartsburd, P.B. \& Labiak, P.H. 2007. Pteridófitas do Parque Estadual de Vila Velha, Ponta Grossa, Paraná, Brasil. Hoehnea 34: 159-209.

Schwartsburd, P.B. \& Labiak, P.H. 2008. Eriosorus areniticola (Pteridaceae), a new species from Brazil. American Fern Journal 98: 160-163.
Schwartsburd, P. B., Labiak, P.H. \& Salino, A. 2007. A new species of Ctenitis (Dryopteridaceae) from southern Brazil. Brittonia 59: 29-32.

Schmitt, J.L., Fleck, R., Burmeister. E.L. \& Rubio, M.A.K. 2006. Diversidade e formas biológicas de pteridófitas da Floresta Nacional de Canela, Rio Grande do Sul: contribuições para o plano de manejo. Pesquisas, Botânica 57: 275-288.

Smith, A.R., Pryer, K.M., Schuettpelz, E., Korall, P., Schneider, H. \& Wolf, P.G. 2008. Fern classification In: T.A. Ranker \& C.H. Haulfer (eds.). Biology and evolution of Ferns and Lycophytes. Cambridge University Press, Cambridge, pp. 417-467.

Steffens, C. \& Windisch, P.G. 2007. Diversidade e formas de vida de pteridófitas no Morro da Harmonia em Teutônia - RS, Brasil. Pesquisas, Botânica 58: 375-382.

Sylvestre, L.S. 1997. Pteridófitas da Reserva Ecológica de Macaé de Cima. In: H.C. Lima \& R.R. Guedes-Bruni (eds.). Serra de Macaé de Cima: Diversidade e Conservação em Mata Atlântica. Instituto de Pesquisas Jardim Botânico do Rio de Janeiro, Rio de Janeiro, pp. 41-52.

Takeda, I.J.M., Moro, R. S. \& Kaczmarech, R. 1996. Análise florística de um encrave de cerrado no Parque do Guartelá, Tibagi, PR. Publicatio 2: 21-31.

Tropicos. 2012. Tropicos. Missouri Botanical Garden. http://www.tropicos.org/ (acesso em 29.12.2012).

UEPG. 2003. Caracterização do Patrimônio Natural dos Campos Gerais do Paraná. Relatório final. Universidade Estadual de Ponta Grossa, Ponta Grossa.

Veloso, H.P., Rangel Filho, A.L.R. \& Lima, J.C.A. 1991. Classificação da vegetação brasileira adaptada a um sistema universal. Fundação Instituto Brasileiro de Geografia e Estatística, Rio de Janeiro.

Ziller, S.R. \& Hatschbach, G. 1996. As formações vegetais do Parque Estadual do Guartelá, Tibagi, PR. Secretaria do Meio Ambiente/Institudo Ambiental do Paraná Curitiba.

Zuloaga, F.O., Morrone, O. \& Belgrano, M.J. 2008. Catálogo de las plantas vasculares del Cono Sur (Argentina, sur de Brasil, Chile, Paraguay y Uruguay). Monographs in Systematic Botany 107: 1-778. 